Anuario del Instituto de Historia Argentina, junio 2018, vol. 18, n 1, e060. ISSN 2314-257X Universidad Nacional de La Plata

Facultad de Humanidades y Ciencias de la Educación

Centro de Historia Argentina y Americana

\title{
Mujeres, profesiones y procesos de profesionalización en la Argentina y Brasil
}

\author{
Biernat, Carolina \\ CONICET, Centro de Estudios en Historia, Cultura y Memoria (CEHCMe) \\ Universidad Nacional de Quilmes, Argentina \\ cbiernat@yahoo.com \\ Queirolo, Graciela \\ Instituto Interdisciplinario de Estudios de Género (IIEGE) \\ Universidad Nacional de Buenos Aires, Argentina \\ graciela.queirolo@gmail.com
}

Cita sugerida: Biernat, C. y Queirolo, G. (2018). Mujeres, profesiones y procesos de profesionalización en la Argentina y Brasil Anuario del Instituto de Historia Argentina, 18 (1), e060. https://doi.org/10.24215/2314-257Xe060 


\section{Mujeres, profesiones y procesos de profesionalización en la Argentina y Brasil}

Womens, professions and professionalizations process in Argentine and Brazil

Carolina Biernat

CONICET, Centro de Estudios en Historia, Cultura y Memoria

(CEHCMe)

Universidad Nacional de Quilmes, Argentina

cbiernat@yahoo.com

Graciela Queirolo

Instituto Interdisciplinario de Estudios de Género (IIEGE)

Universidad Nacional de Buenos Aires, Argentina

graciela.queirolo@gmail.com

Las profesiones desempeñadas por mujeres y los procesos de profesionalización femenina conforman un objeto de análisis de la Historia Social que, en las últimas décadas, viene desplegando un creciente interés. A modo de ejemplo y sin la intención de realizar un examen exhaustivo de esa producción podemos mencionar la publicación de varios dossiers temáticos y libros que las abordan (Frederic, Graciano y Soprano, 2010; Pozzio, 2012; Martin y Ramacciotti, 2016; Correa y Zárate, 2017; Gómez Molla, 2017; Rodríguez y Soprano, 2017; Queirolo y Zárate, en prensa). Se trata de un objeto de estudio construido gracias a un conjunto de desprendimientos de distintos campos historiográficos. Por un lado, el que se produjo desde la Historia Laboral, en cierta medida, gracias a las indagaciones promovidas por la Historia de las Mujeres. En efecto, la búsqueda del protagonismo femenino dentro del mundo del trabajo tempranamente visibilizó la presencia de mujeres en una enorme variedad de ocupaciones que excedían el sector secundario. Junto a las obreras de talleres e industrias y las del trabajo domiciliario se identificó a todas aquellas trabajadoras que se desempeñaban en el sector terciario: desde las enroladas en el sinnúmero de actividades del servicio doméstico -sirvientas y cocineras-, pasando por la heterogénea variedad de empleadas -telefonistas, vendedoras y dactilógrafas-, hasta la inclusión de aquellas vinculadas a las instituciones educativas - maestras y profesorasy a las sociosanitarias -enfermeras, parteras y visitadoras sociales-. El mundo del trabajo urbano de las mujeres se presentó diverso porque la experiencia asalariada reunía actividades que no sólo se desarrollaban en espacios y tiempos laborales particulares, sino que exigían saberes diferentes y formaciones tanto técnicas como profesionales específicas. Por otro lado, los desprendimientos que procedieron de la Historia Social de la Salud y de la Enfermedad allanaron el camino para el análisis de las labores sociosanitarias desde una perspectiva de género, y visibilizaron el proceso de feminización y jerarquización de algunas de estas profesiones a lo largo de la historia. Por último, las nuevas miradas sobre el Estado y la conformación de sus burocracias definieron los interrogantes por las y los expertos que las integraban.

Sin dudas, la pregunta por las mujeres introdujo el análisis de la diferencia sexual, el cual se volvió mucho más profundo cuando se propuso un análisis relacional entre los diferentes géneros -en un primer momento fueron femenino y masculino, pero más recientemente este binarismo se ha complejizado a partir de las sexualidades disidentes- ${ }^{1}$. Esto desplegó varias conclusiones: en primer lugar, así como había muchas ocupaciones protagonizadas por mujeres, también las había protagonizadas por varones; es decir, existía una división genérica de actividades en el mercado laboral. En segundo lugar, la Historia Laboral, incluso la que se había sensibilizado con la perspectiva de género, se había concentrado predominantemente en las ocupaciones industriales, en las extractivas como la minería, en las de transportes como los ferrocarriles y los barcos. Así, las obreras y los obreros habían sido los (y las) protagonistas de sus narrativas. Otras ocupaciones laborales, entre las que se encuentra toda la amplia gama que abordará este dossier, habían sido marginadas del estudio por ser consideradas predominantemente de clase media, o, en el mejor de los casos, habían sido 
abordadas a partir de sus estructuras sindicales, como fue el caso de los empleados -estatales, de comercio, bancarios-.

Las ciencias sociales - la Antropología, la Sociología, la Economía y la Ciencia Política- salieron al auxilio de la Historia Laboral a través de la extensión de la Historia Social y sus desarrollos conceptuales, y porque sugirieron y permitieron una ampliación de la mirada hacia las ocupaciones que excedían el sector obrero. Así, se corroboró que la división genérica del mercado de trabajo manifestó una división patriarcal de las tareas que subordinó a las mujeres a la órbita de los varones (Hartmann, 1994). En esta segregación de los empleos intervino un proceso de construcción social de sentido que definió la feminidad o condición femenina a partir de la maternidad entendida como un hecho biológico. Como las mujeres eran madres poseían naturalmente ciertos atributos emocionales y físicos: la paciencia, la ternura o el amor, la predisposición para tareas rutinarias, la abnegación o el sacrificio. Era esta naturaleza femenina la que se dispensaba dentro del mundo doméstico y les permitía a las mujeres gestionar y ejecutar los múltiples y permanentes quehaceres de la casa -asear, ordenar, abastecer, zurcir, cocinar-, que implicaban asistir y cuidar a otras y otros integrantes del grupo familiar. Así, las mujeres ocuparon empleos en los que desplegaron esa supuesta naturaleza femenina que las hacía eficientes en su desempeño.

El ingreso de las mujeres al mundo del trabajo como un modo de prolongación de su naturaleza femenina vinculada a la identidad maternal trajo consecuencias contradictorias y poco felices. En primer lugar, padecieron un desconocimiento de sus capacidades, predominantemente relacionadas con saberes maternales y domésticos, que ponían en juego para desempeñar sus tareas laborales. En la medida que sus actividades se interpretaron como una transposición de su naturaleza femenina al ámbito laboral no fueron reconocidas como saber hacer específico. Así, una enfermera que tendía las camas de una sala en un hospital sabía hacerlo porque era mujer, no porque lo había aprendido en su casa a partir de repetir las enseñanzas de otra mujer de su grupo familiar. Como demuestra el artículo de Ana Laura Martin, las instituciones sanitarias fueron poco exigentes en la titulación de las enfermeras porque usufructuaron de manera implícita los saberes domésticos que ellas portaban. Esto se interpretó como una descalificación y se manifestó en los niveles salariales reducidos.

En segundo lugar, las mujeres accedieron a procesos de capacitación profesional que ponderaron su naturaleza femenina, lo que le restó mérito a la profesionalización. En ese sentido, Luciana Geroletti retoma la difundida interpretación de que el instinto maternal habilitó a las mujeres para formarse como docentes, mientras que Canela Gavrila destaca que la abnegación femenina les abrió las puertas para ingresar a la profesión de visitadoras sociales. A pesar de la exigencia de las técnicas adquiridas en los procesos de profesionalización que implicaron el pasaje por instituciones de capacitación y la titulación, la condición de experta se desjerarquizó por el vínculo que se le asignó a dichas profesiones con la naturaleza femenina.

Entonces, feminización y profesionalización actuaron como dos fuerzas que se unían y se separaban. Se unían porque la condición femenina, doméstica y maternal permitió a las mujeres el ingreso a ciertos estudios y trabajos. Se separaban, cuando los espacios a los que accedían desestimaban los aprendizajes profesionales al resaltar la naturaleza de la condición femenina.

A medida que las sociedades capitalistas se volvieron más complejas, la salud y las actividades burocráticas exigieron una mano de obra técnicamente capacitada para ejecutar eficientemente las nuevas demandas de cada sector. Como demuestran los artículos que integran el presente dossier, en los sectores señalados se produjo una segregación genérica de los empleos que terminó siendo patriarcal porque ofreció a las mujeres puestos secundarios respecto de los puestos que ofreció a los varones. Parteras, enfermeras, visitadoras de higiene y terapistas ocupacionales no sólo se desempeñaron bajo las estrictas supervisiones de los médicos, sino que los asistieron en sus funciones, mientras que las empleadas bancarias ocuparon los puestos de recepcionistas, secretarias o empleadas de poca jerarquía. Precisamente, fue el proceso de feminización de esas ocupaciones lo que legitimó la posición subordinada. 
Ahora bien, todas ellas, con diferentes temporalidades, integraron procesos de profesionalización que se expresaron en la titulación en el caso de las ocupaciones sociosanitarias citadas, y también de las abogadas, las escribanas y las procuradoras, mientras que las empleadas bancarias debieron rendir pruebas de aptitud para ingresar a sus respectivas instituciones. Cierto es que la capacitación profesional otorgó a sus poseedoras ventajas distintivas respecto de quienes no la poseían y, en ciertos casos, llegó a convertirse en un requisito necesario aunque no suficiente para ejercer la profesión. La idoneidad definitiva marchó de la mano de la condición femenina.

La integración en subordinación de las mujeres al mundo del trabajo se expresó de varias maneras, entre las que se destacó su retribución diferencial. La misma segregación laboral justificó los menores niveles salariales: se trataba de profesiones en las que las mujeres secundaban a varones que tomaban decisiones. El mercado era coherente con las responsabilidades de cada uno. Los procesos de profesionalización no habilitaron los puestos jerárquicos, y cuando lo hicieron, como fue el caso de la titulación universitaria de médicas y abogadas, las mujeres padecieron los obstáculos para su desarrollo profesional. Pero fundamentalmente la capacitación profesional no fue ajena a las nociones de excepcionalidad presentes en todas las ocupaciones del trabajo femenino asalariado, las cuales legitimaron los procedimientos de subordinación. La participación laboral de las mujeres se representó vinculada a la necesidad económica, a la transitoriedad, y como un ingreso que completaba el del marido proveedor (Lobato, 2007). Además, se sumó a las responsabilidades domésticas y de cuidado para provocar las "dobles tareas" que caían sobre tiempos y cuerpos femeninos (Queirolo, 2016).

Cinco artículos componen el dossier. Se trata de trabajos que presentan investigaciones originales que se encuentran en diferentes momentos de su desarrollo, algunos en una fase inicial, otros en proceso, otros ya concluidos. Sin embargo, los une una originalidad que se manifiesta -según cada uno- no sólo en el objeto de estudio -alguno más transitado que otro-, sino, especialmente, en la enorme variedad de fuentes que actúan como evidencia empírica para demostrar las hipótesis propuestas, las cuales, a su vez, también presentan interesantes desafíos metodológicos.

El artículo de Ana Laura Martin compara los procesos de profesionalización de parteras y enfermeras en la ciudad de Buenos Aires entre las últimas décadas del siglo XIX y las primeras del siglo XX. El escrito presenta un contrapunto entre ambas ocupaciones en el que destaca un interés contrapuesto del Estado, personificado tanto en sus agencias -Departamento Nacional de Higiene- como en la Universidad -Facultad de Medicina de la Universidad de Buenos Aires-, que así como insistió por un lado en la profesionalización de la obstetricia, por el otro, desatendió la de la enfermería. Si las parteras se vieron obligadas a diplomarse para ejercer su profesión, las enfermeras pudieron continuar sus actividades de manera empírica. Detrás de estas prácticas, Martin devela que la profesionalización de las parteras se tradujo en una subordinación de su actividad a la supervisión de los médicos, lo que les restó autonomía dentro del campo profesional, mientras que la posición empírica de las enfermeras se sustentó en su condición femenina de aptitud para el cuidado de personas y de asistencia a los auténticos profesionales -los médicos-. La subordinación profesional de las primeras y la devaluación laboral de las segundas relegaron a ambas a posiciones marginales dentro del campo médico que avanzaba velozmente hacia la profesionalización. La autora demuestra que titulación y jerarquización no van siempre de la mano.

Canela Gavrila se ocupa del caso de las visitadoras de Higiene Social entre 1930 y 1950, las precursoras de las actuales trabajadoras sociales. El Estado, representado nuevamente en las universidades nacionales, impulsó el proceso de profesionalización al garantizar la titulación, meta final de los cursos de formación. Su objetivo era crear un cuerpo de "agentes auxiliares de la medicina preventiva", es decir, profesionales que bajo una rigurosa supervisión médica transmitieran a las familias de las clases trabajadoras las condiciones de vida saludables que se expresaban en hábitos de alimentación, limpieza y cuidado de personas. Para semejante proceso de moralización de los sectores populares, las mujeres fueron convocadas a partir de su condición femenina: su abnegación sería clave para la tarea educativa. La hipótesis que demuestra Gavrila es que las visitadoras de higiene se apropiaron de su formación profesional y la transformaron con su práctica. A partir 
del análisis de las conferencias radiales -otro notable hallazgo documental-, las visitadoras interpelaron no sólo a las madres en los procesos de crianza, sino también a los padres. Además denunciaron la necesidad de instituciones estatales que colaboraran con las tareas del cuidado de niñas y niños para aliviar a las madres. Es decir, no reprodujeron acríticamente los saberes aprendidos durante su formación, sino que intervinieron denunciando los excesos que los trabajos reproductivos traían a las mujeres pobres.

Daniela Testa se concentra en las terapistas ocupacionales que integran una profesión que atravesó un particular proceso de profesionalización a partir de la década de 1940. La autora elige desarrollar un contrapunto entre el caso argentino y el caso brasileño. Tanto en uno como en otro, la profesionalización se desencadenó a partir de epidemias de poliomielitis que dejaban visibles secuelas motoras entre sus víctimas. Desde la sociedad civil se crearon instituciones que, si bien en un primer momento promovieron la asistencia a las personas afectadas - muchas veces sus familiares directos-, luego se entrelazaron con el Estado para ofrecer su asesoramiento experto para la creación de instituciones que formaran personal idóneo -diplomado-, reclutado mayoritariamente -aunque no exclusivamente- de la población femenina, para emprender las tareas de rehabilitación. La intervención de organismos internacionales, dentro de un horizonte de ideas desarrollistas, devino clave para propiciar el acercamiento. En el caso de Argentina se trató de la Asociación para la Lucha contra la Parálisis Infantil (ALPI), una institución organizada y gestionada por mujeres de las clases acomodadas, mientras que en el caso de Brasil, se trató de la Asociación Brasilera de Beneficencia para la Rehabilitación de Río de Janeiro (ABBR), una institución organizada por empresarios, pero gestionada por mujeres. Ambas serían centrales para la posterior apertura de la Escuela Nacional de Terapia Ocupacional y de la Escuela de Rehabilitación de la ABBR.

Rosario Gómez Molla aborda la profesionalización femenina en el campo del derecho que originó la formación de tres profesiones: abogadas, escribanas y procuradoras. Se trata de una promisoria investigación emprendida a partir de documentos estatales y universitarios que la autora analiza con una metodología cuantitativa. Entre las fuentes utilizadas se destaca un estudio estadístico elaborado por la Oficina Nacional de la Mujer, que recogió datos sobre la totalidad de los títulos otorgados por las universidades nacionales argentinas en el período 1900-1965. Con este estudio la autora logra visibilizar la expansión de la participación de mujeres en los años sesenta, en un sector universitario poco estudiado por la historiografía preocupada por la formación superior.

Luciana Geroletti presenta su análisis de las empleadas bancarias en Brasil entre 1960 y 1980. Es en esos años cuando los bancos abrieron sus concursos de selección de personal a las mujeres. La autora realiza una interesante comparación con décadas previas, cuando la única opción de profesionalización femenina era el normalismo. Sin embargo, tanto la carrera docente como la carrera bancaria ofrecieron a las mujeres posiciones laborales poco jerarquizadas. En el caso del magisterio, la asimilación de la maestra con la madre ponderó el trabajo como una vocación antes que como una profesión. En el caso del empleo administrativo, las trayectorias laborales finalizaron en posiciones donde primaban las tareas repetitivas por sobre las de toma de decisiones. Esto se tradujo en salarios menores que los salarios de los varones. Asimismo, la doble presencia - productiva y reproductiva- caracterizó esa participación laboral. La construcción de entrevistas orales le permite a la autora recorrer varias trayectorias de empleadas bancarias que se inician con la docencia y saltan al sector bancario, donde finalizan su recorrido profesional.

En suma, a partir de preguntas renovadas, de nuevas y originales fuentes documentales y desde una perspectiva interdisciplinar, este dossier espera hacer un aporte al campo de los estudios de profesiones de mujeres. Por otro lado, si partimos de la premisa de que la investigación en ciencias sociales es a la vez un acto académico y un acto político, el trabajo se propone contribuir a discusiones más generales, como la falta de paridad entre el trabajo femenino y el masculino, las dificultades de acceso de las mujeres a escalafones superiores dentro de su desarrollo profesional, y la no remuneración de las tareas de cuidado. Sólo nos resta agradecer a las autoras de cada artículo por sumarse a la propuesta con entusiasmo y dedicación, ingredientes imprescindibles tanto para lo académico como para lo político. 


\section{REFERENCIAS}

Correa, M. J., y Zárate, M. S. (2017). Historizar la profesionalización sanitaria: perspectivas desde Chile y Argentina. Dynamis, 37(2), 263-272.

Frederic, S., Graciano, O., y Soprano, G. (2010). El Estado argentino y las profesiones liberales académicas y armadas. Rosario: Prohistoria Ediciones

Gómez Molla, R. (2017). Profesionalización femenina, entre las esferas pública y privada. Un recorrido bibliográfico por los estudios sobre profesión, género y familia en la Argentina en el siglo XX. Descentrada, 1(1), 1-9. Recuperado de http://www.descentrada.fahce.unlp.edu.ar/article/view/DESe010

Hartmann, H. (1994 [1976]). Capitalismo, patriarcado y segregación de los empleos por sexos. En C. Borderías, C. Carrasco y C. Alemany (comps.). Las mujeres y el trabajo. Rupturas conceptuales (pp. 253-294). Barcelona: Icaria, FUHEM.

Queirolo, G. (2006). Mujeres que trabajan: una revisión historiográfica del trabajo femenino en la ciudad de Buenos Aires (1890-1940). Novo Topo. Revista de historia y pensamiento critico,3, 29-49.

Queirolo, G. (2016). Dobles tareas: los análisis de Josefina Marpons sobre el trabajo femenino en la década de 1930. Anuario de la Escuela de Historia Virtual,9(7), 81-97.

Queirolo, G., y Zárate, M. S. (libro en prensa). Trabajo, profesionalización y género: recorridos por América Latina (siglos XIX y XX).

Lobato, M. (2007). Historia de las trabajadoras en la Argentina (1869-1960). Buenos Aires: Edhasa.

Martin, A. L., y Ramacciotti, K. I. (2016). Profesiones sociosanitarias: Género e Historia. Avances del Cesor, V. XIII, 15, 81-92.

Pozzio, M. (2012). Análisis de género y estudios sobre profesiones: propuestas y desafíos de un diálogo posible $-\mathrm{y}$ alentador-. Sudamérica, 1, 99-129

Rodríguez, L., y Soprano, G. (ed,) (2017). Profesionales e intelectuales de Estado. Análisis de perfiles y trayectorias en la salud pública, la educación y las fuerzas armadas. Rosario: Prohistoria Ediciones.

\section{Notas}

1 La bibliografía que aborda este recorrido es muy extensa. Una síntesis concisa se encuentra en Barrancos, D. (2008), Mujeres, entre la plaza y la casa; Buenos Aires, Sudamericana, pp. 9-27. 